\title{
Morphological and phylogeographic analysis of Brazilian tortoises (Testudinidae)
}

\author{
T.L. Silva, L.P.R. Venancio and C.R. Bonini-Domingos \\ 2015. Universidade Estadual Paulista, “Júlio de Mesquita Filho”, \\ São José do Rio Preto, SP, Brasil \\ Doctoral thesis, Orienting Prof.: C.R. Bonini-Domingos \\ Corresponding author: T.L. Silva \\ E-mail: lucenabio@hotmail.com
}

Genet. Mol. Res. 14 (4): 18604-18606 (2015)

Received March 2, 2015

Accepted September 29, 2015

Published December 28, 2015

DOI http://dx.doi.org/10.4238/2015.December.28.8

The discriminative potentials of biogeography, vocalization, morphology, cytogenetics, hemoglobin, and molecular profiling of cytochrome $b$ as taxonomic techniques for differentiating Brazilian tortoises were evaluated in this study. In Brazil, two species of tortoises are described, Chelonoidis carbonarius and Chelonoidis denticulatus. However, in the present study, some animals that were initially recognized based on morphological characters and coloring did not correspond to the typical pattern of $C$. carbonarius; these animals were classified as morphotypes 1 and 2. It was proposed that these morphotypes are differentiated species, and they should not be considered as a single taxonomic unit with $C$. carbonarius. Tortoises analyzed were provided by the National Institute for Amazonian Research (INPA); the Emilio Goeldi Museum, PA; municipal zoos in São José do Rio Preto, SP, and Araçatuba, SP; and the Reginaldo Uvo Leone breeding farm for Wild and Exotic Animals, Tabapuã, SP. Based on the data obtained using biogeographic evaluation of specimens in the literature, it was found that $C$. carbonarius is distributed in the Northeast Region of Brazil, and no animal of this pattern was observed in the investigated collections. On the other hand, C. denticulatus is found in all the states of the Legal Amazonia. In addition, isolated individual records of this species exist in the Atlantic Forest in Espírito Santo and Rio de Janeiro and in the Midwest Region composed of the states of Goiás, Mato Grosso, and Mato Grosso 
do Sul. In the Northeast Region, C. denticulatus occurs in the State of Bahia. Morphotype 1 has a wider geographical distribution than $C$. carbonarius, possibly because of several distribution reports associated with $C$. carbonarius, indicating erroneous association of morphotype 1 as a single taxonomic unit with $C$. carbonarius. Morphotype 2 is found only in the states of Pará, Maranhão, and Piauí. These biogeographic data indicate that the distribution of $C$. carbonarius can partially be explained by the fact that all the morphotypes are considered as a single taxonomic unit. Behavioral aspects such as intraspecific communication may be as reliable as morphological or molecular data for inferring evolutionary relationships. Analysis of the physical characteristics of vocalization [fundamental frequency $(\mathrm{Hz})$, interval between notes (s), duration of each note (s), and number of notes from each vocalization] between $C$. carbonarius and morphotype 1 revealed statistically significant differences in the interval between notes $(s)(P=0.0000)$; duration of each note $(\mathrm{s})(P=0.0000)$; frequency of notes $(\mathrm{Hz})(P=0.0009)$; and number of notes $(P=0.0002)$. The results of preference experiments using sound stimulus were inconclusive with respect to speciesspecific vocalization preference; only females of $C$. carbonarius showed intraspecific vocalization preference, indicating possible reproductive isolation mechanisms. To explore the presence of sexual dimorphism and morphological differences between $C$. denticulatus, C. carbonarius, and morphotype 1 , descriptive statistics to analyze the data obtained for the investigated measures were used. Two sets of analysis were conducted - the first for each group, to compare the sexes; and the second for each sex, to compare the groups. To examine the interspecific variation in size and shape, a correlation matrix inprincipal component analysis was used. Next, I used factor analysis to rank the features showing $>0.75$ correlation in the differentiation between the sexes. The results were consistent with the hypothesis that morphotype 1 corresponds to a new species, because it differs from the species pattern in terms of morphology, coloring, and sexual dimorphism. The results of classical cytogenetic analysis - to differentiate $C$. denticulatus, $C$. carbonarius, and morphotype 1 - revealed no consistent data that would enable its use as a taxonomic parameter. Conventional Giemsa staining revealed a diploid chromosome number of $2 n=52$ for all the evaluated groups. The patterns obtained using chromosomal banding techniques showed high similarity and low reproducibility. Moreover, the sensitivity and resolution were insufficient to enable differentiation between the three groups, implying the existence of conserved characteristics of the karyotype in Testudinidae. To establish a hemoglobin profile for $C$. denticulatus, C. carbonarius, and morphotype 1 , and to visualize the hemoglobin fractions of each group, I conducted acid and alkaline electrophoreses. The results of high-performance liquid chromatography revealed percentage differences in the hemoglobin fractions. In addition, electrophoresis of the polypeptide chains under acid and alkaline $\mathrm{pH}$ conditions showed the globin composition of each fraction. The observed differences in the chromatographic profile between C. carbonarius and morphotype 1 with respect to $C$. denticulatus validated the technique as an additional method for elucidating taxonomic issues in Testudinidae. The similarities observed in the hemoglobin profiles of $C$. carbonarius and morphotype 1 suggest recent separation between these groups. Alignment of mitochondrial DNA cytochrome $b$ fragments revealed a degree of homogeneity among the $C$. denticulatus samples and the sequences published in the literature, indicating low genetic variability of the mitochondrial DNA cytochrome $b$ fragment for this species. In contrast, the sequences of the mitochondrial DNA cytochrome $b$ fragments from $C$. carbonarius and morphotypes 1 and 2 differed from those available in the published literature databases, indicating a variable genetic structure. This may be because it does not consider taxonomic divisions within C. carbonarius. Phylogenetic analysis did not reveal an appropriate phylogenetic signal for differentiating $C$. carbonarius and morphotypes 1 
and 2. However, this analysis did differentiate $C$. denticulatus, indicating that the separation of $C$. carbonarius and morphotypes 1 and 2 is more recent than the separation of $C$. denticulatus and C. carbonarius. Owing to the problems associated with the use of cytochrome $b$ in phylogenetic analysis, the polytomy observed for $C$. carbonarius and morphotypes 1 and 2 does not exclude the hypothesis that these samples represent different species. It is possible that the inclusion of morphological, behavioral, and hemoglobin profile data in a mixed matrix with the molecular data would enable the separation of morphotypes 1 and 2 as monophyletic species. This analysis would require data regarding external groups, to facilitate a more robust phylogenetic analysis, thereby enabling greater taxonomic resolution. In view of the differences in the biogeographical pattern, vocalization, specific-sound preference, and morphology found in the present study, I propose that morphotype 1 should be considered a new species. Data regarding the conservation status of natural tortoise populations in Brazil should be reviewed, because of the intensive human pressure on this species. There are no private or public plans for the conservation or recovery of natural tortoise populations. Hence, it is very likely that these animals have a more endangered status than that reported in the literature.

Key words: Chelonoidis; Morphology; Biogeography; Behavior; Hemoglobin; Cytochrome b 